\title{
Impact On Mental Health and Wellbeing in Indigenous Communities Due To Land Loss Resulting From Industrial Resource Development: Protocol for a Systematic Review
}

\section{Nicole Burns}

Wilfrid Laurier University https://orcid.org/0000-0002-6944-5097

Janice Linton

University of Manitoba NJMHS Library: University of Manitoba Neil John Maclean Health Sciences Libraries

Nathaniel Pollock

Memorial University of Newfoundland

Laura Jane Brubacher

UBC: The University of British Columbia

\section{Nadia Green}

University of Alberta Department of Public Health Sciences: University of Alberta School of Public Health

Arn Keeling

Memorial University of Newfoundland

Alex Latta

Wilfrid Laurier University

Jessica Martin

Cornell University

Jenny Rand

Dalhousie University

Melody Morton Ninomiya ( $\triangle$ mmortonninomiya@wlu.ca)

Wilfrid Laurier University https://orcid.org/0000-0002-0196-3410

\section{Protocol}

Keywords: Mental health, resource extraction, industrial development, Indigenous Peoples, land dispossession, systematic review

Posted Date: November 15th, 2021 
DOI: https://doi.org/10.21203/rs.3.rs-1058215/v1

License: (c) (1) This work is licensed under a Creative Commons Attribution 4.0 International License. Read Full License 


\section{Abstract \\ Background}

Indigenous Peoples are impacted by industrial development projects that take place on, or near, their communities. Existing literature on impacts of industrial projects on Indigenous Peoples primarily focus on physical health outcomes and rarely focus on the mental health impacts. To understand the full range of long-term and anticipated health impacts of industrial resource development on Indigenous communities, mental health impacts must be examined. It is well-established that there is a connection between the environment and Indigenous wellbeing, across interrelated dimensions of mental, physical, emotional, and spiritual health. This systematic review will synthesize the evidence on the mental health impacts of land dispossession due to resource extractive projects on Indigenous communities. Looking at the mental health impacts of land dispossession from industrial resource development on Indigenous communities is relevant for a variety of reasons including planning, mitigation strategies, decision making, and negotiations.

\section{Methods}

This review includes an Indigenous Advisory Team and a team of Indigenous and settler scholars. The literature search will use the OVID interface to search Medline, Embase, PsycINFO, and Global Health databases. Non-indexed peer reviewed journals related to Indigenous health or research will be scanned. Books and book chapters will be identified in the Scopus and PsycINFO databases. The grey literature search will also include Google and be limited to reports published by government, academic, and nonprofit organizations. Reference lists of key publications will be checked for additional relevant publications, including theses, dissertations, reports, and other articles not retrieved in the online searches. Additional sources may be recommended by team members. Included documents will focus on Indigenous Peoples in North America, South America, Australia, Aotearoa New Zealand, and Circumpolar regions, research that reports on mental health, and research that is based on land loss connected to dams, mines, agriculture, oil and gas. Literature that meets the inclusion criteria will be screened at the title/abstract and full text stages by two team members in Covidence. The included literature will be rated with a quality appraisal tool and information will be extracted by two team members; a consensus of information will be reached and be submitted for analysis.

\section{Discussion}

The evidence from this review is relevant for land use policy, health impact assessments, economic development, mental health service planning, and communities engaging in development projects.

\section{Systematic review registration:}


Registered in the International Prospective Register of Systematic Reviews (PROSPERO; Registration number CRD42021253720)

\section{Background}

Many industrial resource development projects take place in close proximity to Indigenous communities, and in some cases, on Indigenous treaty lands (1-5). While some Indigenous communities have worked collaboratively with industries and governments to establish sustainable land developments (6), others have experienced non-collaborative processes which have resulted in land dispossession through project construction or community relocation $(5,7)$. Several studies of land dispossession have reported on negative health outcomes for Indigenous Peoples (8-10). Assessing the expected and potential health impacts of land loss through industrial development can provide Indigenous communities, industries, and governments with evidence that is necessary for planning, negotiation, mitigation, and monitoring.

There is a substantial body of knowledge on the occupational and community health consequences associated with industries such as mining, energy, and agriculture $(7,10-14)$. However, two recent scoping reviews $(7,10)$ highlight limited evidence about the mental health impacts of resource extraction industries. Most studies have focused on physical health outcomes and industry workers, with comparatively few investigations of mental health or with Indigenous populations $(7,10)$.

Understanding the possible impacts of industrial resource development on mental health in Indigenous communities requires a recognition of the connection between the environment and Indigenous health and wellbeing $(15,16)$. The relationship between Indigenous Peoples and land is part of a holistic ontology that situates mental health as interconnected with all other dimensions of health (physical, emotional, and spiritual) and with cultural identity (16-21). Given these links, industrial resource development that has physical impacts on Indigenous lands and territories, changes access to landbased activities, or results in community displacement, may serve as unique pathways for mental health risks and outcomes in Indigenous communities.

\section{Objective and Question}

The objective of this systematic review is to examine and synthesize the evidence related to the mental health impacts of industrial resource development on Indigenous communities, with a specific focus on consequences due to land loss. This review will answer the question: What are the impacts of land loss due to industrial resource development on mental health and wellbeing in Indigenous communities?

\section{Methods}

This protocol was registered in the International Prospective Register of Systematic Reviews (Registration number CRD42021253720) and has been prepared in accordance with the statement for Preferred Reporting Items for Systematic Review and Meta-Analysis Protocols (PRISMA-P) $(22,23)$. The completed PRISMA-P Checklist is available as Additional File 1. 
We will conduct a systematic review of the peer reviewed and grey literature on the impact of land loss related to industrial resource development on mental health and wellbeing in Indigenous communities. This protocol was developed by an interdisciplinary team of Indigenous and settler (non-Indigenous) scholars with expertise in Indigenous health and wellbeing, environmental stewardship, mental health, resource extraction, and Indigenous People's health literature databases. In keeping with principles and practices for community-based participatory (24) and Indigenous research (25-29), this review was designed with support from a community advisory team, comprised of four Indigenous and one settler members. Their role was to advise on the research objective and question, the inclusion and exclusion criteria, and the information that we will extract from included studies. This approach is founded on the ethical imperative of "nothing about us without us" in Indigenous research (16, 26, 30-32). In this case, the review was requested so that the evidence can support mental health and wellness planning, decision-making, and programming.

\section{Search Strategy}

An academic health sciences librarian with over twenty years of experience working in Indigenous health will develop the search strategy. In consultation with team members, keywords and topics have been translated to the appropriate subject headings. Test searches have been carried out in Scopus and Medline. The OVID interface will be used to search Medline, Embase, PsycINFO, and Global Health.

Several peer reviewed journals related to Indigenous health or research will be scanned or hand searched by members of the team to identify potentially relevant articles. These journals have been selected because they have not been indexed consistently in the biomedical databases. This will ensure comprehensiveness in identifying all available literature and ensuring that scholarly articles written by Indigenous researchers have not been overlooked.

Books and book chapters will be identified in the Scopus and PsycINFO databases and through federated searches in inter-institutional academic library catalogues. Grey literature searches will be carried out using advanced search techniques in Google. Grey literature will be limited to reports or research published by government, academic, and non-profit organizations. Reference lists of key publications will be checked for additional relevant publications, including theses, dissertations, reports, and other articles not retrieved in the online searches. Additionally, sources that our team are aware of pertaining to land loss and mental health will be recommended if they are not captured in the other searches.

Detailed search strategies for searching Medline and Scopus are included in the supplementary material (Additional File 2). Results from the database searches will be imported into Covidence, an application designed for conducting systematic reviews. After duplicate results are removed, we will have two team members complete the title and abstract screening and full text screening for sources that meet our inclusion criteria.

We will include studies focused on three concept areas: (1) Indigenous Peoples in global regions with similar histories of colonization (North America, South America, Australia, Aotearoa New Zealand, and 
Circumpolar regions); (2) mental health risks and outcomes; and (3) land loss connected to specific resource extraction industries/sectors (hydro-electric dams, mining, agriculture, oil and gas). Table 1 describes a detailed version of our inclusion criteria. We will have two independent reviewers complete the extraction and quality appraisal phase. The extracted data will be compared side-by-side to reach consensus. Any disagreements between reviewers at any stage of the review will be discussed with a third reviewer until an agreement is made.

Table 1

Inclusion Criteria and Rationale

\section{Inclusion Criteria}

Printed in English.

Reporting on studies that include Indigenous groups or communities.

Research focuses on Indigenous Peoples within North America, South America, Australia, New Zealand, and Circumpolar Regions

Research reports on mental health risk/protective factors, experiences, outcomes, and/or impacts.

Research is based on land development, extractive industries, and/or contamination leading to loss of access to land from dams, mines, agriculture, oil and gas.

Full text is available.

Must be a primary study.

\section{Rationale}

Nobody on the team is fluent in another language and able to review the documents in the time available.

We are excluding any studies that are focused on only one person or self-reporting.

Indigenous Peoples in the listed areas share commonalities in the history of colonization.

The focus of this systematic review is to examine how loss of access to land impacts Indigenous Peoples' mental health so, it is imperative that the studies included report on this element.

We are focusing on the four major land development industries that result in loss of access to land. We are including contamination that leads to the loss of access to land but not focusing on the biomedical results from contamination that may also be connected to mental health.

The full text must be available for appraisal and analysis to be included.

We will exclude all discussion papers, literature reviews, and commentaries for analysis; however, we will read relevant documents as it may inform the background literature and discussion of findings.

\section{Quality Appraisal}

We are adapting questions in the Critical Appraisal Skills Programme (33) qualitative research appraisal tool to be inclusive of quantitative and mixed research methodologies and methods. We will not exclude studies based on research methodologies and methods. Collectively, the co-authors have expertise in quantitative, qualitative, and mixed research methodologies. We will weight evidence in the review based on quality. That is, studies that score high using the appraisal assessment tool, will be emphasized in the results. We anticipate that there will be limited number of studies that meet the inclusion criteria, so we do not plan to exclude studies based on low scores. In addition to changing the questions in the appraisal 
tool to be inclusive of quantitative and qualitative studies (Question 2 below), we added a relevance question to assess whether a direct connection is made between losing/decreasing access to land and mental health in each study (Question 10 below). The questions in the appraisal tool focus on the quality, rigor, and relevance of the study and include:

1. Was there a clear statement of the aims of the research?

2. Is the methodology appropriate?

3. Was the research design appropriate to address the aims of the research?

4. Was the recruitment strategy appropriate to the aims of the research?

5 . Was the data collected in a way that addressed the research issue?

6. Has the relationship between researcher and participants been adequately considered?

7. Have ethical issues been taken into consideration?

8. Was the data analysis sufficiently rigorous?

9. Is there a clear statement of findings?

10. How clear is the connection between losing access to land and mental health made in the study?

\section{Data Extraction}

The following information will be extracted from each included study.

1. Reference (Authors, Date, Title)

2. Type of Study (article, report, other)

3. Geographical Location(s)

4. Indigenous Peoples (Nations, Groups, Organizations)

5. Level of IP involvement in governing research (e.g., initiating the research and identifying priority, guidance and governance on research methodology, gathering data, analysis, how research findings are used, and document writing.)

6. Type of development/industry (dam, mining, agriculture, or oil/gas)

7. Research Question(s)

8. Study Design/Methodology (qualitative, quantitative, mixed methods)

9. Research Methods Used

10. Tools and other indicators to assess, measure, document mental health and wellness

11. Reported mental health impacts and outcomes

12. Recommendations

\section{Data Analysis}

The extracted data will be exported into an Excel spreadsheet and shared with the full team of authors. We will hold meetings to discuss the overarching findings as a large group and identify a sub-group to immerse themselves in conducting a thorough data analysis. The sub-group will be comprised of at least three team members who will independently use an iterative and inductive thematic coding process to 
analyze the reported mental health impacts and outcomes. Having two or more coders is shown to improve rigour, reliability, and accountability (34). The sub-group will meet weekly to share and discuss thematic analyses until consensus is reached. The full team will meet to review the preliminary results, provide further input and feedback, and co-develop figures that summarize the reported findings. Our results will be compared with other literature related to key themes from this review.

\section{Discussion}

This systematic review will assess and synthesize the evidence on the mental health impacts of resource development projects that lead to land loss for Indigenous communities. This will include an examination of potential direct and indirect effects. This evidence will be relevant to stakeholders involved and invested in industrial resource development and land use policy, health impact assessments, economic development, and mental health service planning. Potential knowledge users of evidence from this review include Indigenous communities, various levels of government, public health professionals, and industry.

\section{Strengths And Limitations}

The primary strength of this review is the collective diverse expertise within our team, which helped to inform the protocol and will aid in data analysis and understanding; this includes having an experienced librarian with expertise in Indigenous health that can develop a comprehensive search strategy. Some anticipated limitations include the potentially limited number of eligible studies. Previous scoping reviews with similar search strategies identified very few studies that report on Indigenous Peoples' mental health in connection to extractive industries $(7,10)$. We are focusing the review on four major resource development industries but recognize that other related industries that may also report on the mental health impacts of Indigenous Peoples. Finally, by restricting the publication language to English, we may limit the number of papers from South America in particular.

\section{Declarations}

\section{Author Contributions}

NB drafted this manuscript with significant content input from JL, NP, and MMN. JL led the development of the search strategy. MMN is responsible for the systematic review and facilitating the collaborative work with the community partner. All authors contributed to the design of the review, and revised and approved the manuscript.

\section{Acknowledgements}

We would like to acknowledge and thank the Advisory Team.

\section{Funding}


The funding to support this research is provided by West Moberly First Nations. The Advisory Team for this systematic review is primarily comprised of West Moberly First Nations members who were involved in defining the research question and the scope of literature. The Advisory Team and funder were not involved in the literature search, data collection, analysis, interpretation, or writing of this manuscript.

\section{Competing Interests}

The senior author (MMN) received funding from WMFN to conduct the systematic review; some coauthors were hired on a contract basis through this funding. There is nothing to be gained or lost based on the findings from this review, now or in the future, by any of the co-authors.

\section{Ethics approval}

This research does not involve human subjects. It is exempted from research ethics board review.

\section{Consent for publication}

Not applicable

\section{Availability of data and material}

Not applicable

\section{References}

1. Brisbois B, Feagan M, Stime B, Paz IK, Berb Es-Blázquez M, Gaibor J, et al. Mining, colonial legacies, and neoliberalism: A political ecology of health knowledge. A J Environ Occup Heal Policy. 2021;31(1):48-64.

2. Government of Canada. Indigenous Mining Agreements. Natural Resources Canada. 2021.

3. Hipwell W, Mamen K, Weitzner V, Whiteman G. Aboriginal peoples and mining in Canada: Consultation, participation and prospects for change. Ottawa North-South Inst. 2002;10.

4. Keeling A, Sandlos J. Mining and communities in Northern Canada: History, politics, and memory. Vol. 3. University of Calgary Press; 2015.

5. Sandlos J, Keeling A. Aboriginal communities, traditional knowledge, and the environmental legacies of extractive development in Canada. Extr Ind Soc. 2016;3(2):278-87.

6. Rodon T. Institutional development and resource development: The case of Canada's Indigenous peoples. Can J Dev Stud Can d'études du développement. 2018;39(1):119-36.

7. Brisbois BW, Reschny J, Fyfe TM, Harder HG, Parkes MW, Allison S, et al. Mapping research on resource extraction and health: A scoping review. Extr Ind Soc. 2019 Jan 1;6(1):250-9.

8. Reid J, Varona G, Fisher M, Smith C. Understanding Maori 'lived' culture to determine cultural connectedness and wellbeing. J Popul Res. 2016 Mar 19;33(1):31-49. 
9. Whitbeck L, Adams G, Hoyt D, Chen X. Conceptualizing and measuring historical trauma among American Indian people. Am J Community Psychol [Internet]. 2004 Jun [cited 2021 Oct 25];33(34):119-30. Available from: https://pubmed.ncbi.nlm.nih.gov/15212173/

10. Myette E, Riva M. Surveying the complex social-ecological pathways between resource extraction and Indigenous Peoples' health in Canada: A scoping review with a realist perspective. Extr Ind Soc. 2021;100901.

11. Fernández-Llamazares Á, Garteizgogeascoa M, Basu N, Brondizio ES, Cabeza M, Martínez-Alier J, et al. A state-of-the-art review of indigenous peoples and environmental pollution. Integr Environ Assess Manag. 2020;16(3):324-41.

12. Cortes-Ramirez J, Naish S, Sly PD, Jagals P. Mortality and morbidity in populations in the vicinity of coal mining: a systematic review. BMC Public Health. 2018;18(1):1-17.

13. Gamu J, Le Billon P, Spiegel S. Extractive industries and poverty: A review of recent findings and linkage mechanisms. Extr Ind Soc. 2015;2(1):162-76.

14. Hendryx $M$, Ahern MM. Relations between health indicators and residential proximity to coal mining in West Virginia. Am J Public Health. 2008;98(4):669-71.

15. Fast E, Lefebvre M, Reid C, Deer WB, Swiftwolfe D, Clark M, et al. Restoring Our Roots: Land-Based Community by and for Indigenous Youth. Int J Indig Heal. 2021 Jan;16(2).

16. King M, Smith A, Gracey M. Indigenous health part 2: the underlying causes of the health gap. Lancet [Internet]. 2009;374. Available from: http://dx.doi.org/10.1016/S0140-6736(09)60827-8

17. Berry HL, Bowen K, Kjellstrom T. Climate change and mental health: a causal pathways framework. Int J Public Health. 2010;55(2):123-32.

18. Cunsolo A, Ellis NR. Ecological grief as a mental health response to climate change-related loss. Nat Clim Chang. 2018;8(4):275-81.

19. Gracey M, King M. Indigenous health part 1: Determinants and disease patterns. Lancet. 2009;374(9683):65-75.

20. Greenwood ML, de Leeuw S, Lindsay NM, Reading C. Determinants of Indigenous Peoples' Health in Canada: Beyond the Social. Toronto: Canadian Scholars' Press; 2015.

21. Reading C, Wien F. Health inequities and the social determinants of Aboriginal Peoples' health [Internet]. 2009 [cited 2021 Oct 25]. Available from: .

22. Moher D, Shamseer L, Clarke M, Ghersi D, Liberati A, Petticrew M, et al. Preferred reporting items for systematic review and meta-analysis protocols (PRISMA-P) 2015 statement. Syst Rev 2015 41. 2015 Jan;4(1):1-9.

23. Shamseer L, Moher D, Clarke M, Ghersi D, Liberati A, Petticrew M, et al. Preferred reporting items for systematic review and meta-analysis protocols (PRISMA-P) 2015: elaboration and explanation. BMJ [Internet]. 2015 Jan 2 [cited 2021 Nov 1];349. Available from: https://www.bmj.com/content/349/bmj.g7647 
24. Israel BA, Schulz AJ, Parker EA, Becker AB. Community-based participatory research: policy recommendations for promoting a partnership approach in health research. Educ Health (Abingdon) [Internet]. 2001 [cited 2021 Oct 26];14(2):182-97. Available from: https://pubmed.ncbi.nlm.nih.gov/14742017/

25. Jacklin K, Kinoshameg P. Developing a Participatory Aboriginal Health Research Project: "Only if it's Going to Mean Something." J Empir Res Hum Res Ethics. 2008;3(2):53-67.

26. First Nations Information Governance C. OCAP: Ownership, control, access and possession. Centre FNIG, editor. Vol. 2019. Ottawa; 2018.

27. Inuit Tapiriit K, Nunavut Research I. Negotiating Research Relationships with Inuit Communities: A Guide for Researchers [Internet]. Nickels S, Shirley J, Laidler G, editors. Ottawa and Iqaluit; 2007. Available from: https://www.itk.ca/sites/default/files/Negotitiating-Research-RelationshipsResearchers-Guide.pdf

28. Kovach M. Indigenous methodologies: Characteristics, conversation, and contexts. Toronto: University of Toronto Press; 2009. 201 p.

29. Smith LT. Decolonizing methodologies: Research and Indigenous peoples. New York: Zed Books Ltd.; 2012. $256 \mathrm{p}$.

30. Bull J, Beazley K, Shea J, MacQuarrie C, Hudson A, Shaw K, et al. Shifting practise: Recognizing Indigenous rights holders in research ethics review. Qual Res Organ Manag An Int J. 2019 Oct;15(1):21-35.

31. Huria T, Palmer SC, Pitama S, Beckert L, Lacey C, Ewen S, et al. Consolidated criteria for strengthening reporting of health research involving indigenous peoples: the CONSIDER statement. BMC Med Res Methodol 2019191 [Internet]. 2019 Aug 9 [cited 2021 Oct 26];19(1):1-9. Available from: https://bmcmedresmethodol.biomedcentral.com/articles/10.1186/s12874-019-0815-8

32. Jones J, Cunsolo A, Harper SL. Who is research serving? A systematic realist review of circumpolar environment-related Indigenous health literature. PLoS One [Internet]. 2018 May 1 [cited 2021 Nov 1];13(5):e0196090. Available from: https://journals.plos.org/plosone/article? id=10.1371/journal.pone .0196090

33. Critical Appraisal Skills Programme UK. CASP CHECKLISTS - CASP - Critical Appraisal Skills Programme [Internet]. [cited 2021 Nov 4]. Available from: https://casp-uk.net/casp-tools-checklists/

34. Cornish F, Gillespie A, Zittoun T. Collaborative analysis of qualitative data. Sage Handb Qual data Anal. 2013;79:93.

\section{Supplementary Files}

This is a list of supplementary files associated with this preprint. Click to download.

- MedlineandScopussearchstrategies.doc

- PRISMAPchecklistBurnsLinton.docx 